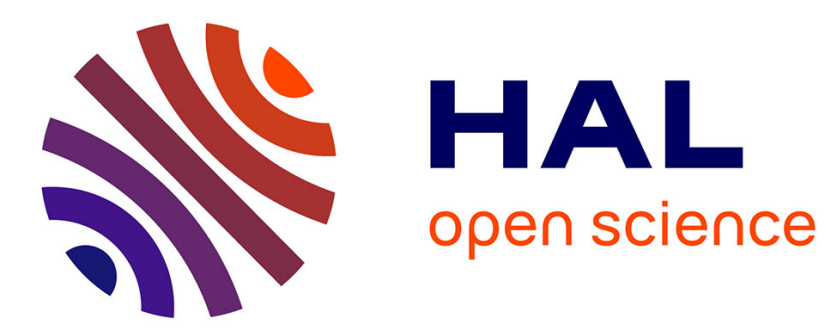

\title{
Photoinduced charge-transfer in chromophore-labeled gold nanoclusters: quantum evidence of the critical role of ligands and vibronic couplings
}

Adrian Domínguez-Castro, Carlos R Lien-Medrano, Khaoula Maghrebi, Sabri Messaoudi, Thomas Frauenheim, Arnaud Fihey

\section{To cite this version:}

Adrian Domínguez-Castro, Carlos R Lien-Medrano, Khaoula Maghrebi, Sabri Messaoudi, Thomas Frauenheim, et al.. Photoinduced charge-transfer in chromophore-labeled gold nanoclusters: quantum evidence of the critical role of ligands and vibronic couplings. Nanoscale, 2021, 13 (14), pp.6786-6797. 10.1039/d1nr00213a . hal-03193290

\section{HAL Id: hal-03193290 \\ https://hal.science/hal-03193290}

Submitted on 23 Jun 2021

HAL is a multi-disciplinary open access archive for the deposit and dissemination of scientific research documents, whether they are published or not. The documents may come from teaching and research institutions in France or abroad, or from public or private research centers.
L'archive ouverte pluridisciplinaire HAL, est destinée au dépôt et à la diffusion de documents scientifiques de niveau recherche, publiés ou non, émanant des établissements d'enseignement et de recherche français ou étrangers, des laboratoires publics ou privés. 


\title{
Journal Name
}

\section{ARTICLE TYPE}

Cite this: DOI: $00.0000 / x x x x x x x x x x$

\section{Photoinduced Charge-Transfer in Chromophore-Labeled Gold Nanoclusters: Quantum Evidence of the Critical Role of Ligands and Vibronic Couplings $\dagger \ddagger$}

\author{
Adrian Domínguez-Castro, ${ }^{a}$ Carlos R. Lien-Medrano, ${ }^{* a}$ Khaoula Maghrebi, ${ }^{b, c}$ Sabri \\ Messaoudi, $, c, d$ Thomas Frauenheim, ${ }^{* e, f, a}$ and Arnaud Fihey*
}

Received Date

Accepted Date

DOI: $00.0000 / x x x x x x x x x x$

The electron flow between a metallic aggregate and an organic molecule after excitation with light is a crucial step on which are based the hybrid photovoltaic nanomaterials. So far, designing such device with the help of theoretical approaches have been heavily limited by the computational cost of quantum dynamics models able to track the evolution of the excited states over time. In this contribution we present the first application of Time-Dependent Density Functional Tight-Binding (TD-DFTB) method for an experimental nanometer-sized gold-organic system consisting in a hexylprotected $\mathrm{Au}_{25}$ cluster labelled with a pyrene fluorophore, in which the fluorescence quenching of the pyrene is attributed to an electron transfer from the metallic cluster to the dye. The full quantum rationalization of the electron transfer is attained through quantum dynamics simulations, highlighting the crucial role of the protecting ligands shell in the electron transfer, as well as the coupling with nuclear movement. This work paves the way towards a fast and accurate theoretical design of optoelectronic nanodevices.

\section{Introduction}

Noble metal nanoclusters stand as highly versatile building blocks for nanomaterials, between the molecular world and solid state systems, with a deep structure-properties relationship. 1 They can be used as grafting support, similarly as for functionalized nanoparticles, but possess a highly symmetric arrangement a discrete electronic structure and an important electronic gap, granting them a molecular-like behavior. Among them, a large number of gold nanoclusters (GNC) have been synthesized and characterized up to today, ranging from $\mathrm{Au}_{13}$ to $\mathrm{Au}_{329} \cdot \frac{2+6}{6}$ With the in-

\footnotetext{
${ }^{a}$ Bremen Center for Computational Materials Science (BCCMS), Universität Bremen, Germany; E-mail: cmedrano@uni-bremen.de

${ }^{b}$ Faculty of Sciences of Bizerte FSB, Carthage University, 7021 Jarzouna, Tunisia

${ }^{c}$ Laboratory of Materials, Molecules and Applications, IPEST, Carthage University, Sidi Bou Said Road, B.P. 51 La Marsa- 2070, Tunisia

'Department of Chemistry, College of Science, Qassim University, Buraidah 51452, Saudi Arabia

${ }^{e}$ Beijing Computational Research Center (CSRC), Beijing, China; E-mail: frauenheim@csrc.ac.cn

${ }^{f}$ Shenzen JL Computational Science and Applied Research Institute (CSAR), Shenzhen, China

${ }^{g}$ Univ Rennes, CNRS, ISCR (Institut des Sciences Chimiques de Rennes) - UMR 6226, F-35000 Rennes, France; E-mail: arnaud.fihey@univ-rennes1.fr

\$ This article is dedicated to Professor Thomas Frauenheim, a special mentor and friend, on the occasion of his 70th birthday

$\dagger$ Electronic Supplementary Information (ESI) available: [details of any supplementary information available should be included here]. See DOI: 00.0000/00000000.
}

crease in nanocluster size can be observed the progressive shift of the electronic and optical properties from a discrete behavior, 2,4 to a nearly metallic structure with the appearance of a localized surface plasmon resonance (LSPR) band. ${ }^{[5]}$ They have been considered the last ten years as potential solar energy harvester, ${ }^{7} \mathrm{es}$ pecially when the coat of organic ligands around the cluster not only protects the metal but also possesses photochemical properties. In the case of chromophore-labelled nanoclusters, one can expect an electron flow to occur between the two parts after excitation with light. Such excited-state electron transfer may induce sufficient charge separation for them to be collected in a photovoltaic device. A few systems composed of a nanocluster functionalized with photoactive molecules (photochromes and fluorophores) can be found in the literature. $\frac{8-10}{10}$ For instance, a coat of azobenzene photochromes have been linked to a $\mathrm{Au}_{25} \mathrm{GNC}$, allowing the organic shell to be collectively switched by light between a -cis and -trans conformations, impacting the properties of the cluster in return. The exchange ligand reaction used to functionalized the GNC allows to finely control the number of chromophores to be attached. $\frac{9] 10}{1 n}$ the case of fluorophore compounds, a $\mathrm{Au}_{38}$ GNC have been decorated with one, two or three porphyrins, $\frac{10}{10}$ and a single pyrene derivative has been grafted to a $\mathrm{Au}_{25}$ GNC.9 In this last study, the quenching of the pyrene fluorescence is attributed, based on spectroscopic evidences, to an electron transfer from the GNC to the excited molecule. Such hypothesis is yet to be confronted to excited state quantum mod- 
eling. Indeed, theoretical description of such hybrid gold organic phototactive compounds remains today a challenge for quantum chemistry; the large number of electrons in the system limits the use of high-level ab-initio methods, and even Density Functional Theory (DFT) and its Time-Dependent (TD-DFT) counterpart become hardly tractable for most of the functionalized GNCs. If DFT studies have been conducted on some of these hybrid systems to rationalize their electronic and vertical optical behavior, $\frac{1112}{112}$ the prediction of the excited state evolution through DFT electron dynamics remains limited to small gold cluster without any protecting ligands $\sqrt{13}$ or grafted chromophores.14 $\frac{16}{16}$ Up to today, the quantum investigation of excited-state electron transfer in larger gold organic nanomaterials, like ligands protected $\mathrm{Au}_{25} \mathrm{SR}_{18}$ (composed of hundreds of atoms) have been unreachable.

In this context, the Density Functional Tight Binding (DFTB) scheme, 17 an approximated DFT method where the tabulation of DFT integrals prior to calculations allows to reduce by two or three order of magnitude the computational cost, is a tool of choice for large scale systems. The DFTB + package is an opensource implementation of the DFTB method developed by our group. Since its original release in 200718 , several extensions were added to the package, and it now encompasses the most popular features of DFT. ${ }^{19}$ For instance, the Time-Dependent DFTB (TD-DFTB) allows to explore excited-state properties in the Casida's formalism, 20 and in the real time domain. ${ }^{21}$ The latter can be used to access not only the absorption spectrum but the electron dynamics following excitation.22 This method was succesfully used in the last decade to study the optoelectronic properties of a wide variety of organic and inorganic materials: the absorption spectra and photophysical properties of photosyntetic pigments ${ }^{2324}$ and DNA-protected silver emitters ${ }^{25 \mid 26}$, plasmonic properties of gold, $27-29$ silver ${ }^{28 \mid 29}$ and aluminium 30 metallic nanoparticles, optical properties of carbon-based materials like graphene nanoflakes ${ }^{31}$ and graphene nanoribbons ${ }^{32}$ and photoinduced charge transfer mechanisms elucidation in metal oxides ${ }^{33-37}$ for dye-sensitized solar cells (DSSC) and molecular aggregates ${ }^{3839}$ for organic solar cells (OSC). These studies were performed within the electron-only quantum dynamics approach without considering nuclei movement, yet reaching a good agreement with experiments.

In the last years, evidence that the nuclei movement is also of key importance to study charge transfer processes below the $100 \mathrm{fs}$ scale has appeared. Experimental and theoretical studies showed that quantum-coherent motion of nuclei and electrons promote coherent charge oscillations, a crucial step in the rearrangement and stabilisation of charge separation. Such process has been observed in covalently ${ }^{40}$ and non-covalently ${ }^{41 / 42}$ linked organic systems and vertically stacked transition-metal dichalcogenides ${ }^{43 / 44}$. These works clearly highlights the need of taking into account the nuclei movement to accurately describe charge transfer processes in systems where the coherent vibronic coupling can play a fundamental role at early states. Regarding this point, the implementation of the semiclassical Ehrenfest method for nuclear-electron dynamics in DFTB+ allow us to consider the nuclei motion within the TD-DFTB approach. This method has already been successfully used to study coherent breathing oscillations on metal nanoparticles, 45 transient absorption spectra in organic molecules, ${ }^{46}$ impulsive vibrational spectroscopy on biological systems, 47 and photoinduced charge transfer in sensitized nanodiamonds. 22

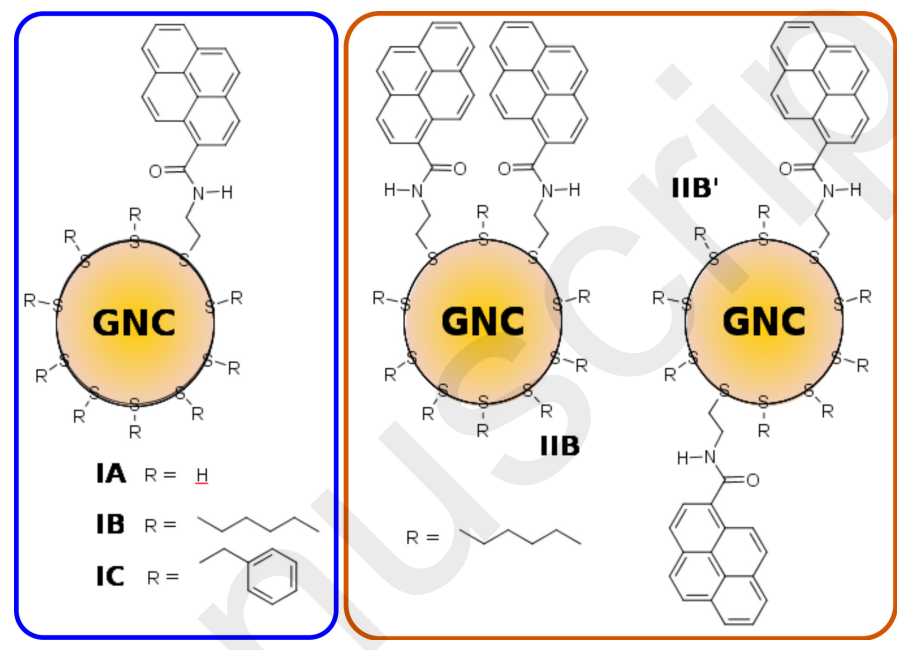

Fig. 1 Schematic representation of the systems studied in the present work. On the left side, $\left[\mathrm{Au}_{25}(\mathrm{SR})_{17} \mathrm{SPyr}\right]^{-}$, i.e., one pyrene molecule attached to the gold nanocluster $\left[\mathrm{Au}_{25}(\mathrm{SR})_{18}\right]^{-}(\mathrm{GNC})$ with different protecting groups $\mathrm{R}$ : $\mathrm{H}$, hexyl and phenylethyl for IA, IB and IC, respectively. On the right side, two newly designed systems with pyrene molecules attached in two different positions.

In a experimental work of M.S. Devadas et al. ${ }^{9}$ was highlighted the ability of a $\mathrm{Au}_{25}$-based GNC to act as an electron donor. Using electrochemical and ultrafast time-resolved fluorescence and absorption techniques, they probed the interfacial photoinduced charge transfer processes between the GNC and a fluorescent pyrene derivative ligand, within 580 fs. Certainly, this type of photoinduced events opened the doors to design a new generation of nano-scale sensors, catalysts, and light harvesting systems. 9 Yet, no atomic understanding of such complex charge transfer process has been proposed yet, and this study propose to fill this blank.

After a validation of the DFTB description of the ground-state (geometry, electronic structure) and UV-Visible absorption features of $\left[\mathrm{Au}_{25}\left(\mathrm{SC}_{6} \mathrm{H}_{13}\right)_{18}\right]^{-}$GNC and its pyrene-labelled counterpart $\left[\mathrm{Au}_{25}\left(\mathrm{SC}_{6} \mathrm{H}_{13}\right)_{17} \mathrm{SPyr}\right]^{-}$(IB in Fig. 1 and see as well Fig. S1 for a more detailed scheme of the substitution positions), we detail the potential electron transfer occurring after light irradiation within the real-time TD-DFTB electron dynamics scheme. The amount and direction of the charge transfer is described, and the role of the aliphatic protecting ligands is unraveled. The effect of temperature is also taken into account by coupling the electron dynamics to a molecular dynamics step allowing to select a representative batch of conformations. Based on this observations, we finally propose new hybrid systems with potential superior electron transfer ability. 


\section{Computational methods}

The electronic structure of the ground state (GS) in all studied system was obtained using the self-consistent-charge densityfunctional-tight-binding (SCC-DFTB). 48 B0 Based on a controlled approximation of Density Functional Theory (DFT), this method has been succesfully used to describe the electronic structure of large biological and organic systems as well as their quantum properties. 50 51 Specifically, all the DFTB calculations were performed using the DFTB + code,$\frac{52}{5 i}$ with the auorg set of parameters ${ }^{53}$ in the second-order SCC-DFTB. Ground-state structures of all systems were obtained with a geometry optimization with a force criterion of $10^{-4}$ au, followed by a vibrational frequencies calculation to ensure that the structures correspond to a global energy minimum.

The optical properties were simulated through the TD-SCCDFTB method, which is an extension of the SCC-DFTB method to the time domain, and it is already implemented on DFTB+. In this method, an initial perturbation in the shape of a Dirac delta pulse $\left(\hat{H}=\hat{H}^{0}+E_{0} \delta\left(t-t_{0}\right) \hat{\mu}\right)$ is applied to the initial groundstate density matrix $(\hat{\rho})$. After the pulse application, $\hat{\rho}$ evolves in time and its evolution can be resolved by time integration of the Liouville-von Newmann equation of motion (EOM):

$$
\frac{\partial \hat{\rho}}{\partial t}=-\frac{i}{\hbar}\left(S^{-1} \hat{H}[\hat{\rho}] \hat{\rho}-\hat{\rho} \hat{H}[\hat{\rho}] S^{-1}\right),
$$

where $\hat{\rho}$ is the single electron density matrix, $S$ is the overlap matrix and $\hat{H}$ is the SCC-DFTB Hamiltonian.

By Fourier-transforming the dipole moment signal in the time domain and dividing by the Dirac-delta-like pulse field strength the polarizability tensor can be calculated:

$$
\alpha(\omega)=\frac{\mu(\omega)}{E_{0}}
$$

For the systems under analysis in the present paper, we propagated 82684 steps using a time step of $0.0024 \mathrm{fs}$ and an electric field intensity of $E_{0}=0.001 \mathrm{VA}^{-1}$, well within the linear response regime.

In the case of the photoinduced charge transfer dynamics, non-adiabatic molecular dynamics were performed within the Ehrenfest approximation. In this approximation the electrons are treated as quantum and nuclei as classical particles that move in a mean field caused by the instantaneous electronic wave-function. The new equation of motion of the $\hat{\rho}$ is similar to the Liouville-von Neumann equation (eq 1) but including non-adiabatic coupling terms $D$ :

$$
\dot{\hat{\rho}}=-\frac{i}{\hbar}\left(S^{-1} \hat{H}[\hat{\rho}] \hat{\rho}-\hat{\rho} \hat{H}[\hat{\rho}] S^{-1}\right)-\left(S^{-1} D[\hat{\rho}] \hat{\rho}-\hat{\rho} D^{\dagger}[\hat{\rho}] S^{-1}\right)
$$

The first term of the right-hand side of this equation recovers the electronic EOM of eq 1 and it has been successfully used for the calculation of optical properties and photoinduced charge transfer phenomena keeping the nuclei "clamped".23|31|54|58 The second term gives rise to the non-adiabatic interactions, allowing energy exchange between electrons and nuclei at the Ehrenfest level. The perturbation added to the Hamiltonian and used to study the photoinduced charge transfer processes takes now the shape of a $\sin ^{2}$ pulse, simulating the experimental laser pulse. A deeper explanation of the implementation and scope of this method is described in ref 22 . In this work, an electric field strength of $0.1 \mathrm{~V} / \AA$ and an energy laser of $400 \mathrm{~nm}$ (expressed in $\mathrm{eV}$ units equal to 3.0996) were used. The total duration of the pulse was $100 \mathrm{fs}$ in all cases.

The molecular dynamics presented in this work were performed using Bohr-Oppenheimer MD simulations in the DFTB+ framework on the basis of an NVT assemble. ${ }^{52}$ A total of 25 ps were simulated. The equation of motion is integrated using the Velocity Verlet algorithm, with a time step of 0.25 fs. Temperature was set by a linear increasing during the first 1000 steps and controlled at $\mathrm{T}=300 \mathrm{~K}$, using Andersen thermostat.

Reference DFT calculations presented in this paper were obtained with the PBE functional, associated to a 6-31G(d,p) basis set for C, N, O, S and H atoms and a LANL2DZ basis set and pseudopotential for $\mathrm{Au}$ atoms. The Gaussian 16 software ${ }^{59}$ was used for these calculations. $\frac{59}{59}$

\section{Results and discussion}

\subsection{DFTB Ground state description.}

The auorg set of parameters have been developed more specifically to describe gold bulk and surfaces or slabs decorated with organic molecules, $[53$ and has yet to be tested on GNCs. As a matter of fact, these GNCs possess a specific core-shell atomic arrangement, e.g., for $\mathrm{Au}_{25}(\mathrm{SR})_{18}^{-}$, the icosahedral cluster core encompasses 13 gold atoms and its outer part is build with 6 $\mathrm{S}-\mathrm{Au}-\mathrm{S}$-Au-S staples. This core-shell arrangement is retrieved in DFTB, and Table 1 compares the average Au-Au and Au-S distances within the GNC obtained with DFTB/auorg to a DFT reference calculation and to the X-ray crystallographic data. ${ }^{3}$ The $\mathrm{S}-\mathrm{Au}-\mathrm{S}$ and Au-S-Au angles are found similar to the references, while the DFTB accuracy for the bond distances varies depending on the bond type. Au(core)-Au(core) and Au(shell)-S(shell) mean distances are in acceptable agreement with the experimental structure. The former bond type is underestimated by $4 \%$ and the latter is overestimated by $3 \%$, compared to XR data.

$\mathrm{The} \mathrm{Au}$ (core)-Au(shell) and $\mathrm{Au}$ (core)-S(shell) mean distances are more drastically overestimated in DFTB, with a $12 \%$ and $8 \%$ deviation to XR data, respectively. This exaggerate further the tendency of the full DFT calculation to overestimate these distances. These deviations mainly impact the general structure of the GNC by expanding the core-shell distance.

It should be noted that DFT calculations are conducted with a model compound, $\mathrm{Au}_{25}(\mathrm{SH})_{18}^{-}$, where the aliphatic chains are replaced by simple $\mathrm{H}$ atoms due to computation limitations. DFTB results are presented in the case of this same model system, and for the $\mathrm{Au}_{25}\left(\mathrm{SC}_{6} \mathrm{H}_{13}\right)_{18}^{-}$system without any truncation. The impact of the presence of the hexyl chains on the cluster geometry is trifling, with 0.01 to $0.04 \AA$ change in the distances, and less than $3^{\circ}$ variation in the angles. Exchanging one the chain with a pyrene derivatives to form compound IB (see Fig. 1) do not impact further the geometry of the GNC.

The electronic structure of the $\mathrm{Au}_{25}(\mathrm{SH})_{18}^{-}$GNC is analyzed by plotting the density of states (DOS) in Fig. S2, with contribu- 


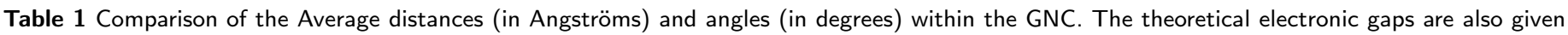
(in eV)

\begin{tabular}{|c|c|c|c|c|}
\hline & DFTB $\left(\mathrm{Au}_{25}(\mathrm{SH})_{18}^{-}\right)$ & DFTB $\left(\mathrm{Au}_{25}\left(\mathrm{SC}_{6} \mathrm{H}_{13}\right)_{18}^{-}\right)$ & DFT $\left(\mathrm{Au}_{25}(\mathrm{SH})_{18}^{-}\right)$ & Exp. 3 \\
\hline$\overline{\mathrm{Au}(\text { core)-Au(core) }}$ & 2.77 & 2.78 & 2.96 & 2.88 \\
\hline Au(shell)-S(shell) & 2.38 & 2.39 & 2.38 & 2.31 \\
\hline Au(core)-Au(shell) & 3.53 & 3.49 & 3.29 & 3.16 \\
\hline $\mathrm{Au}$ (core)-S(shell) & 2.57 & 2.60 & 2.48 & 2.37 \\
\hline S-Au-S & 177.7 & 174.2 & 172.7 & 172.3 \\
\hline $\mathrm{Au}-\mathrm{S}-\mathrm{Au}$ & 103.5 & 101.3 & 101.5 & 100.9 \\
\hline Electronic gap & 1.22 & 1.13 & 1.34 & - \\
\hline
\end{tabular}

tions of the $s p$ and $d$ orbitals separated for $\mathrm{Au}$ and $\mathrm{S}$ atoms, and compared to a DFT reference. A global agreement is found between DFTB and the reference DOS, especially around the electronic gap, while higher non-occupied states are less accurately reproduced. The electronic HOMO-LUMO gap of the bare GNC, $\mathrm{Au}_{25}(\mathrm{SH})_{18}^{-}$, is found to be $1.22 \mathrm{eV}$, close to the DFT value of $1.34 \mathrm{eV}$. Adding the $\mathrm{C}_{6} \mathrm{H}_{13}$ ligands in the DFTB model slightly decreases this value, down to $1.13 \mathrm{eV}$. The effects of the protecting ligands shell on the electronic structure consists mainly in a shift of the overall DOS, as can be seen in Fig. S3 of the Supporting Information. Finally, the DOS of the experimental pyrenelabelled system, IB, projected on the GNC, the hexyl ligands and the pyrene (Fig. S4) allows to rationalize the electronic interactions between these different elements at the DFTB level and to compare them to a DFT reference. Such decomposition indicates that the electronic states of the IB compound is a simple addition of the different contributions from its constituting parts, without new delocalized states. In both DFTB and DFT, the highest occupied level is located on the GNC while the first empty level is found on the Pyrene unit.

\subsection{Ground State Absorption Spectra.}

Fig. 2a represents the corresponding absorption spectra obtained for the IA $(\mathrm{R}=\mathrm{H})$ system and its constituting parts. Two main features appear: i) a high absorption band at 400nm (matching with the characteristic peak of pyrene in UV-region), and ii) the total absorption spectra for the IA system can be interpreted as the sum of the individual absorption spectra of the GNC and the pyrene. These findings are in agreement with the work of Dedavas et al., 9 and show that there are no interactions in the ground state between those parts (also supported by the DOS presented in Fig. S4). Fig. 2b displays the absorption spectra for IB $\left(R=\mathrm{C}_{6} \mathrm{H}_{13}\right)$. It can be observed a slight red-shift of the GNC absorption bands (comparing with system IA in Fig. 2a) but the main features of the absorption spectrum are not affected, displaying an intense absorption band in the UV-region with a maximum peak located at $400 \mathrm{~nm}$. A identical conclusion can be drawn for IB: the total absorption spectra is still the sum of the individual absorption spectra of the GNC and pyrene systems (hexyl groups do not present absorption bands in the region plotted).

The experimental maximum excitation band, involving the pyrene moiety, is found at $345 \mathrm{~nm}, 9$ while in the TD-DFTB absorption spectrum, this peak is found at $400 \mathrm{~nm}$. Such bathochromic shift remain acceptable, and may originate from: the lack of solute-solvent interactions $\sqrt{60}$, since the simulation was performed in gas phase at $0 \mathrm{~K}$, and more importantly from the type of density functional used in the DFTB parameterization. Indeed, as shown in a previous DFT study of this system, 11 the optical properties of such $\pi$-conjugated chromophore are more accurately reproduced when using a more sophisticated functional including a range-separation term, while a pure GGA functional returns bands too low in energy. This is then somewhat expected for auorg set of parameters, created with a GGA type of functional, 53 to inherit such errors. Switching to a recent DFTB model to construct the parameters (not yet available for $\mathrm{Au}$ and $\mathrm{S}$ atoms) based on a range-separated type of functional, 61 may correct this behavior. Concerning the absorption profile of the GNC moiety (in red in Fig. 2), the global shape of the spectrum is found in agreement with both experimental results and TD-DFT studies. $\frac{3 \mid 11}{}$ Particularly, the low energy band in the near infra-red region, and the two intense bands between $400 \mathrm{~nm}$ and $500 \mathrm{~nm}$, characteristic of this GNC, are correctly reproduced. Overall, these observations demonstrate the ability of our TD-DFTB method to reproduce the absorption spectra of the experimental hybrid systems of interest.

\subsection{Charge transfer dynamics.}

Fig. 3 shows for systems IA $(R=H)$ and IB $\left(R=\mathrm{C}_{6} \mathrm{H}_{13}\right)$ the evolution of the Mulliken charge distribution on the GNC and the pyrene derivative (including the $-\mathrm{CH}(\mathrm{O}) \mathrm{NHCH}_{2} \mathrm{CH}_{3}$ linker) upon photoexcitation with a $100 \mathrm{fs}$ width pulsed electric field perturbation, in tune with the transition dipole moment of the pyrene excitation at $400 \mathrm{~nm}$ (in Fig. S5 is represented the transition dipole moment). These Ehrenfest dynamics were performed at 0 K.

In both cases, it can be observed an ultrafast photoinduced charge transfer within the first $100 \mathrm{fs}$ (pulse width) of the dynamics. The GNC $\Delta q$ curve takes positive values (i.e, loss of electrons), while the pyrene curve takes negative values (gain of electrons). These results confirm the charge transfer and the electron donor behaviour of the GNC previously described by Devadas and coworkers in this system. 9

During the dynamics of IA, the charges separation starts to decrease after $300 \mathrm{fs}$, while the IB system reaches a stationary charge-separated state (at least within the time window investigated). The presence of the hexyl chains induce then a clear stabilization of the charge transferred after excitation, suggesting that they play an important role in the charge transfer mechanism. To illustrate this role, Fig. 4 represents isosurfaces of the charge density difference (CDD) computed by subtracting the electron density at $160 \mathrm{fs}$ respect to the initial time, at $0 \mathrm{fs}$. By this defi- 


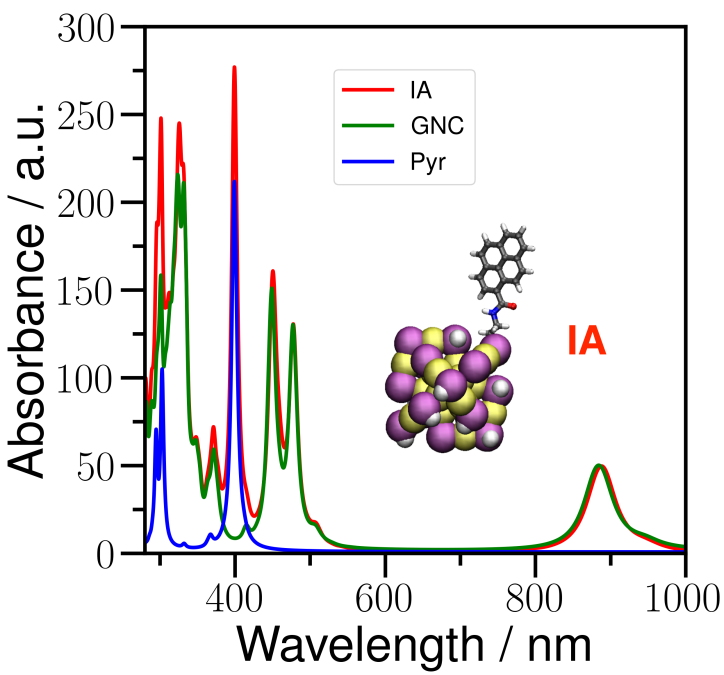

(a)

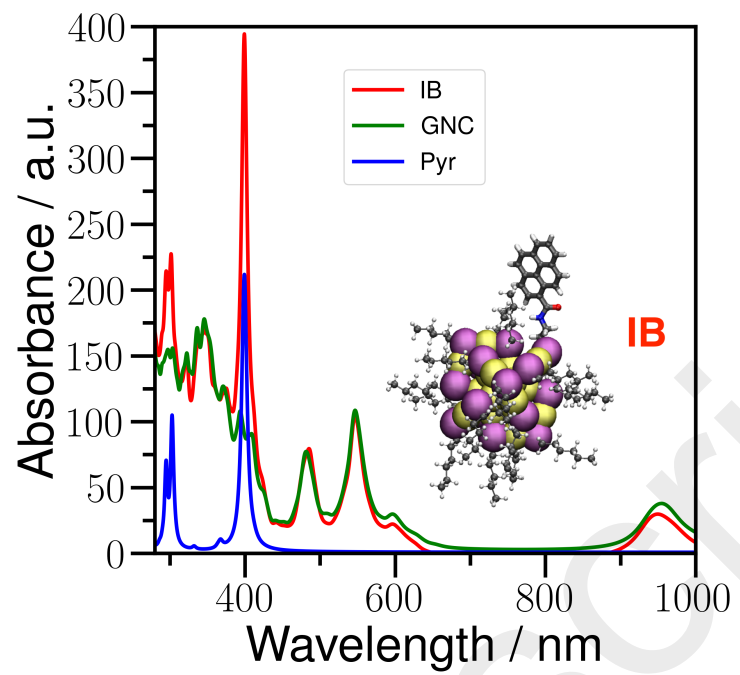

(b)

Fig. 2 (a) Absorption spectra for IA system. (b) Absorption spectra for IB system (Gold, Sulfur, Carbon, Oxygen, Nitrogen, Hydrogen atoms are represented in yellow, purple, gray, red, blue and white, respectively).

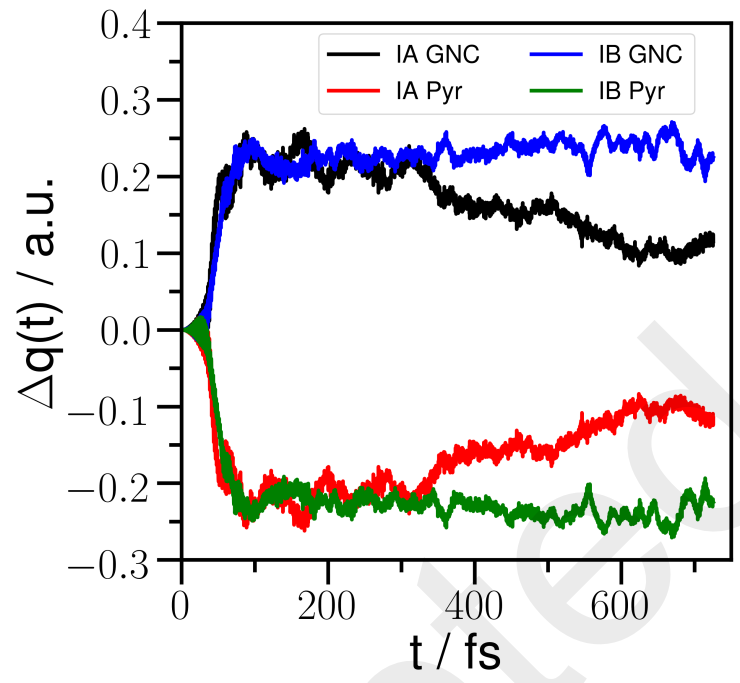

Fig. $3 \Delta q$ as a function of time for IA and IB systems under study.

nition, a positive area (green isosurface) appears when the electronic density is more negative at the time of $160 \mathrm{fs}$, traducing a charge accumulation. A negative area (orange isosurface) corresponds to a more positive electronic density at the time of 160 fs, i.e., a charge depletion. For IA (Fig. 4a), one can find in the region corresponding to the linker between the pyrene unit and the GNC a depleted region, while the accumulation of charge is mainly localized on the pyrene unit. Noteworthy, small accumulation regions can also be found on the gold atoms of the external shell of the cluster, in accordance with their previously observed role in oxidation processes. ${ }^{12|62| 63}$ The CDD analysis of IB (Fig. $4 \mathrm{~b}$ also shows an accumulation region on the pyrene, but large depleted regions are now found on the hexyl ligands. Even if the reader should keep in mind that the simplistic picture of the CDD is not directly to be compared to the whole dynamic behavior described previously, these plots illustrate the acceptor character the pyrene after irradiation in both cases, and, more importantly highlights the role played by the protecting ligands in IB, as the main electron donor in the charge-transfer mechanism.

\subsubsection{Effects of nuclear movements and temperature in the charge-transfer process.}

Fig. 5 shows the evolution of the Mulliken charge distribution in the GNC with and without alkyl ligands (IA and IB, respectively) upon the photoexcitation of the pyrene molecule in the same conditions as mentioned before. The green and blue curves represent the dynamics considering clamped and moving ions, respectively. In the case of system IA (Fig. 5a)with simple $\mathrm{H}$ as protecting ligands, the nuclear motion does not seem to affect the charge transfer mechanism since the two curves presents similar behaviour. The same conclusion can be drawn in the case of IB (Fig. 5b) substituted with hexyl chains. In this case however, if both curves show the same trend and describe the same mechanism, the nuclear motion decreases the total steady-state charge transferred by half, since a new relaxation channel (i.e., the nuclear degrees of freedom) was added. This increases the detuning between the electronic states responsible of the charge transfer, and allows reaching the steady-state sooner, as previously observed in a nanodiamond/dye complex. 32

To take into account the effect of the temperature, Ehrenfest dynamics were conducted starting from decorrelated configurations sampled at $300 \mathrm{~K}$ from an NVT ensemble using BornOppenheimer molecular dynamics (see supporting information for more details). Fig. 5 also shows the mean charge of the GNC (donor) obtained from averaging the Ehrenfest dynamics for 25 decorrelated configurations, together with its standard deviation. Clearly, within the configurations sampled, the charge transfer always occurs denoting the robustness of the process and it does not depend on a certain molecular geometry. Comparing the mean curve at $300 \mathrm{~K}$ with the clamped and moving ions 


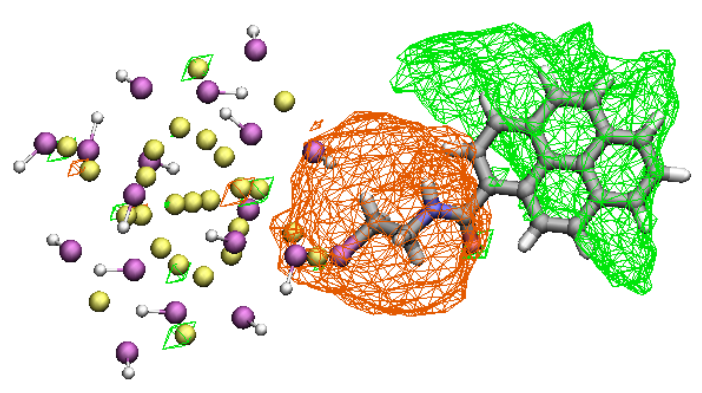

(a)

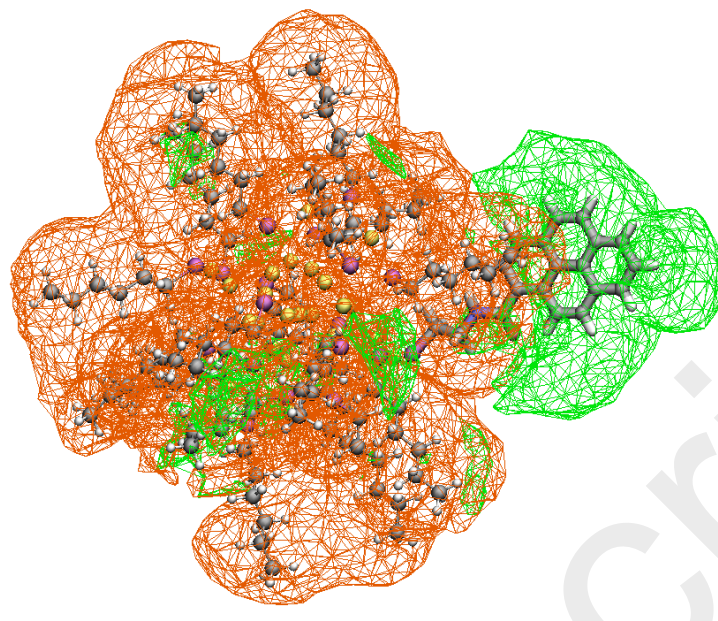

(b)

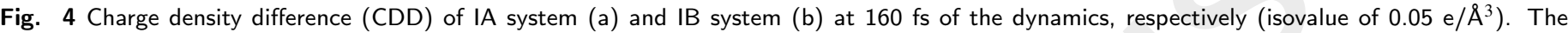

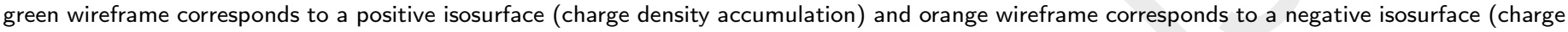

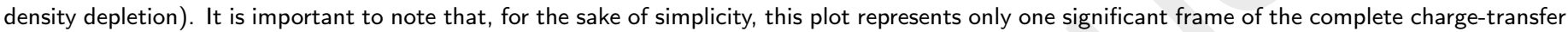
dynamic, avoiding the complexity of the dynamical oscillations in the CDD produced as a natural consequence of the pulse perturbation used.

curves at $0 \mathrm{~K}$ in Fig. $5 \mathrm{a}$ one can observed lower charge values within the time window simulated, showing that the addition of temperature, in the case of IA, decreases the amount of charge transferred. Interestingly, the same is not observed in the case of system IB (Fig. 5b). In this case, taking into account the temperature in the simulation increases on average the amount of charge transferred when comparing with the moving ions curve at $0 \mathrm{~K}$. Particularly, the difference between both curves is bigger at early stages (between 100 and $400 \mathrm{fs)} \mathrm{of} \mathrm{the} \mathrm{dynamics.} \mathrm{The}$ charge transfer enhancement mediated by temperature suggest a non-trivial contribution of the nuclei movement at the very first steps of the process, and only when the GNC is protected with alkyl ligands.

\subsection{Ligands-mediated charge transfer mechanism for IB}

To unravel the higher charge transfer ability in IB and get more insight about the role of the ligands, Fig. 6 6a and 6b compares the charge transfer (from one conformation of the sampling at $300 \mathrm{~K}$ ) for IA and IB, and for the latter the contribution of the donor is split in two: the hexyl chains and the GNC core. This new analysis highlights the clear donor role of the hexyl chains (with a large amount of charge donated), and also surprisingly shows that in IB, the GNC core starts to act as an electron donor at the very beginning of the dynamic, before accepting electrons from the hexyl chains (see inset from Fig. 6b). We propose then the following three-steps mechanism to explain the electron transfer to the pyrene in the system IB:

- a preparation step, up to approximately $30 \mathrm{fs}$ where the dye is being excited,

- a second step where a small amount of charge is transferred directly from the GNC core to the pyrene residue (between 30 and $100 \mathrm{fs}$ ), followed by,

- a rapid compensation (starting around $100 \mathrm{fs}$ ) where the alkyl ligands stabilize the charge, with a transfer to the GNC and pyrene residue.

The third step, clearly reveals the important role of the protecting ligands giving the cluster its donor capacity proposed and observed by Dedavas et al. ${ }^{9}$ These observations and the mechanism proposed can also be observed for the Ehrenfest dynamic at $0 \mathrm{~K}$ (from Fig. 3) when the different contributions of the GNC and ligands are separated (see Fig. S14).

Another interesting feature of the dynamics presented in Fig. $6 \mathrm{a}$ and $6 \mathrm{~b}$ is the presence of oscillations in the curves in the scale of a few tens of femtoseconds. Following the strategy of Rozzi 40 and Falke ${ }^{41}$ to elucidate the nature of such oscillations, Fig. $6 \mathrm{c}$ and $6 \mathrm{~d}$ present the Fourier intensity spectrum of the simulated charge transfer dynamics for IA and IB, respectively. For IB appears a strong signal about $\sim 1235 \mathrm{~cm}^{-1}$, associated with the typical C-C stretching mode $\left(\sim 1130 \mathrm{~cm}^{-1}\right)$ of the hexyl chains. For IA can also be found a less intense peak at $\sim 1255 \mathrm{~cm}^{-1}$. In the absence of protecting hexyl chains, this peak is attributed to the $\mathrm{C}-\mathrm{C}$ stretching mode of the linker group of the Pyrene derivative. In addition, for IB, can be observed an intense peak around 280 $\mathrm{cm}^{-1}$, most likely related to Au-S stretching modes and Au-S-C bending modes (showing the coupling between the core and the alkyl chains), and some other peaks between $600-800 \mathrm{~cm}^{-1}$, that could correspond to C-S stretching modes. 64,67

These differences suggest a charge transfer mechanism mediated by vibronic couplings, that are increased in the presence of the ligands, highlighting further the role of the alkyl chains. The charge transfer process not only promotes coherent oscillations mainly by the C-C, Au-S and Au-S-C vibrational modes of the system from the very early stages but also is enhanced under the addition of temperature where this modes are already excited when the Ehrenfest dynamic starts (as shown in Fig. 5). 


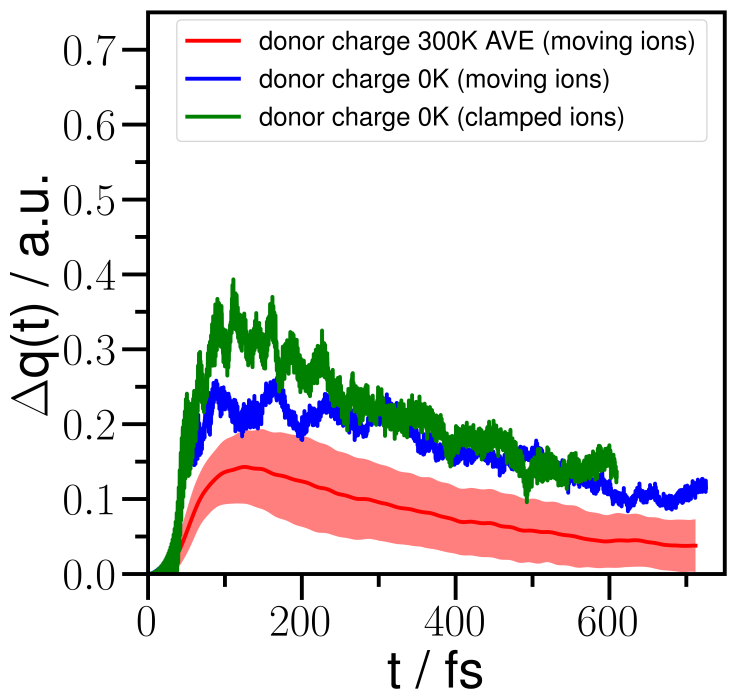

(a)

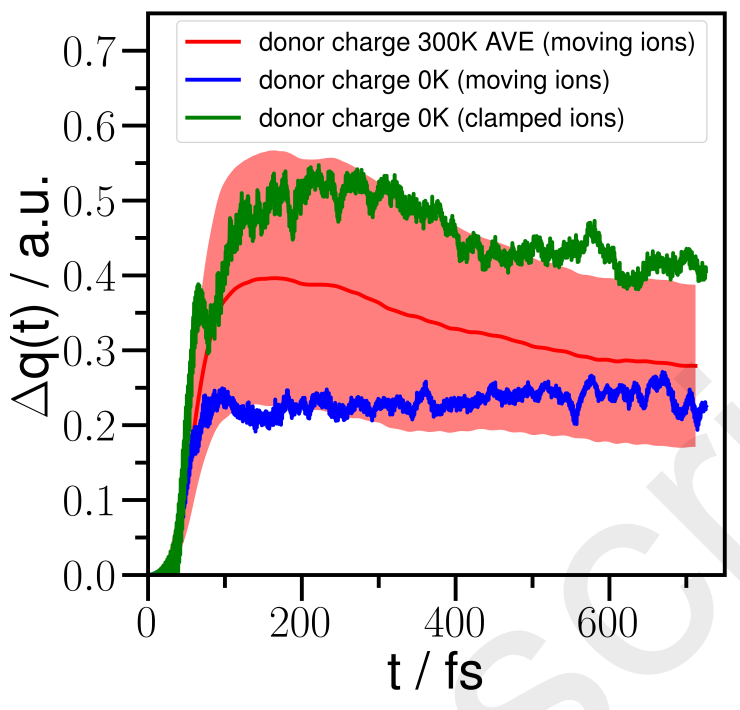

(b)

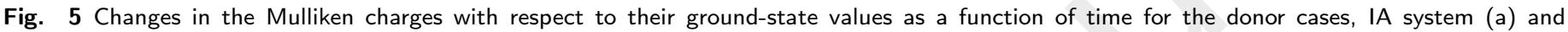

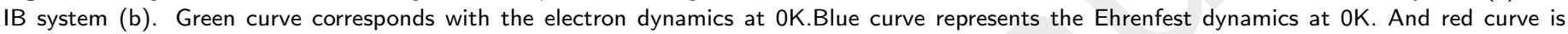

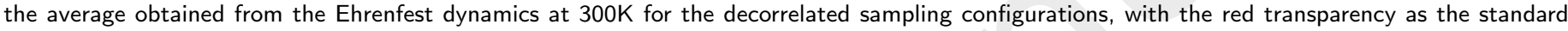
deviation obtained, respectively, for each case under study.

\subsection{Influence of the nature of the protecting ligands.}

An efficient photoinduced charge transfer is clearly observed in IB due to remarkable role of the protecting ligands, and such role should not be overlooked when designing GNC-based candidate for charge transfer processes. Based on this conclusions, we will now discuss another scenario where the $\mathrm{C}_{6} \mathrm{H}_{13}$ protecting units are replaced with an other type of ligands, in order to achieve a more complete understanding of the effect of functionalization of the GNC on the photoinduced charge transfer. $\mathrm{Au}_{25}$ family of gold nanoclusters can indeed be synthesized with various protecting groups. 1 Among those, this section is dedicated to the comparison of system IB to an homologuous compound, IC, possessing phenyl-ethyl (PhEt) units as ligands, very common in GNC synthesis procedures. 3

The incorporation of the PhEt ligands does not affect the main features of the absorption spectrum already described in the case of IB (see Fig. S15). Notably, the intense absorption band of the pyrene is retrieved identical, at $400 \mathrm{~nm}$, in IC, hinting that there are no ground-state interactions with the GNC. Fig. 7b displays the changes in the Mulliken charges with respect to their groundstate values as a function of time for IC, after an excitation at 400 $\mathrm{nm}$, along with those of IB for comparison purpose. For the new IC system, an ultrafast charge transfer is observed, from the GNC to the pyrene residue, with an stable charge separation after 100 fs. Overall, the charge transfer profile obtained with an Ehrenfest dynamics performed at $0 \mathrm{~K}$ is thus similar to the one described previously for IB in the same conditions. Important differences appear when separating the PhEt ligands contribution to $\Delta q$ from the contribution of the GNC core (Fig. 7c). If in IB and IC the two types of protecting ligands act as electron donors in the transfer, it is clear that the PhEt units exhibit a less important $\Delta q$ compared to the $\mathrm{C}_{6} \mathrm{H}_{13}$ chains (Fig. 6b). Furthermore, the mechanism in the charge transfer clearly differs from the one established for IB in the previous section. In this case, one can see that both the GNC and the PhEt ligands directly donate charge to pyrene acceptor, along the whole dynamic.

While ultimately, the efficiency of the charge transfer process is not affected by the nature of the protecting ligands around the GNC (for both cases is obtained a comparable charge transfer, in term of amount of charge transferred and stabilization of this charge separation), it is highly noticeable that the GNC core presents an overall acceptor character when attached to aliphatic chains, but acts as an electron donor when protected with Phenylterminated ligands. This comfort the fact that designing such photoactive systems can not be done without an explicit consideration of the whole ligands shell in the model.

\subsection{Influence of the number of chromophores.}

In this type of hybrid systems, it is relatively straightforward to control the number of chromophores anchored to the GNC, by adjusting the pyrene:GNC ratio during the ligand exchange reaction. 10 In this section, a GNC functionalized with two pyrene residues is considered, and the impact of such fictionalization on the charge transfer is evaluated. To this end, two different conformations are created (see Fig. $7 \mathrm{~d}$ blue for a 3D representation, and Fig. S1 for a more detailed scheme of the substitution pattern), that correspond to a scenario where the two pyrene derivatives are either placed on neighbor sulfur anchors (IIB) or on opposite sites (IIB'). The absorption spectra for IIB and IIB' are displayed in Fig. S15 of the Supporting Information. In both cases, the most intense absorption band in the wavelength range of interest is again the unshifted pyrene band located at $400 \mathrm{~nm}$, as no groundstate interactions occurs with the GNC. Compared to the absorption profile of $I B$, the incorporation of a second pyrene residue 


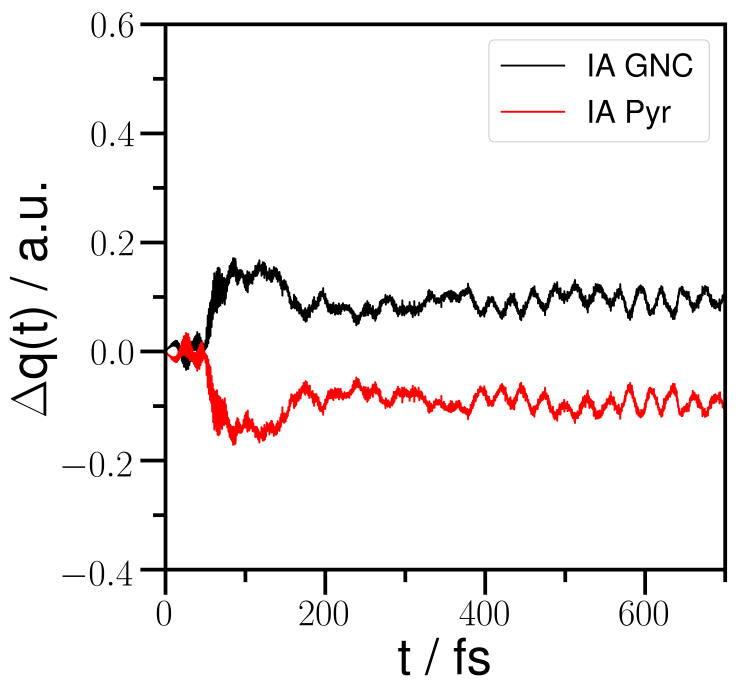

(a)

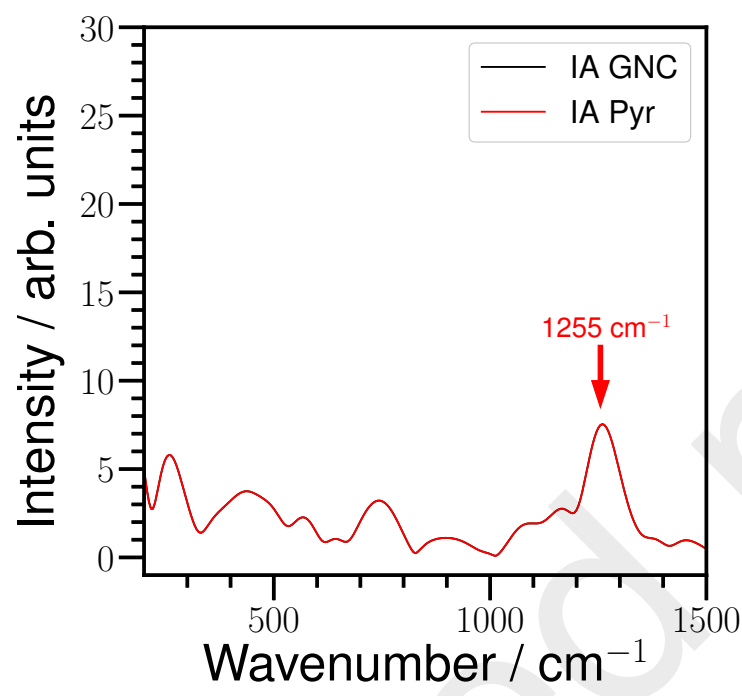

(c)

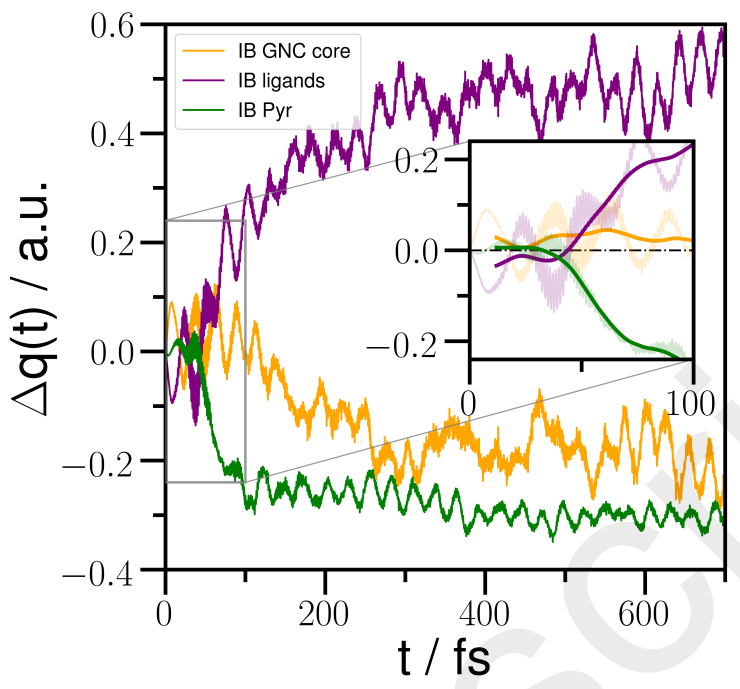

(b)

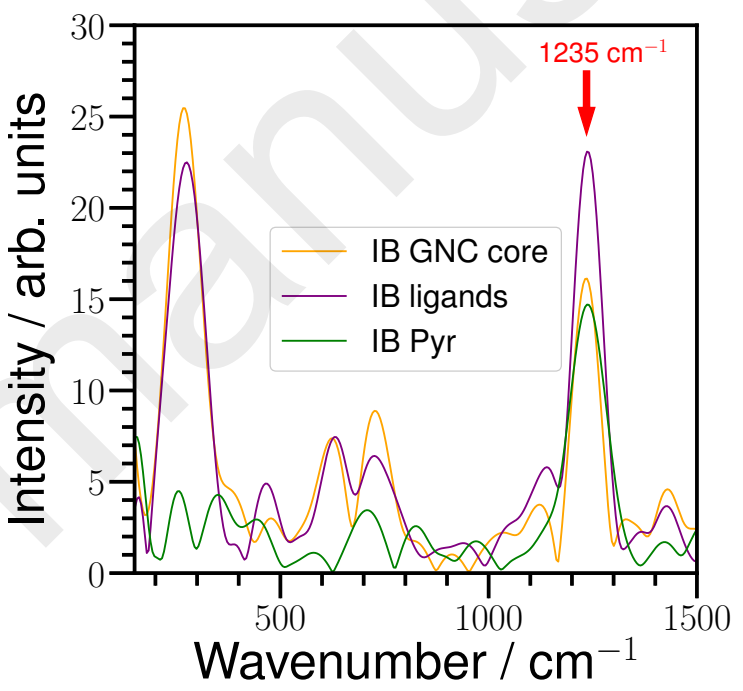

(d)

Fig. $6(\mathrm{Up}) \Delta \mathrm{q}$ as a function of time for one single frame from the NVT assemble presented in Fig. 5 for IA (a) and IB (b). (Bottom) Fourier intensity spectrum of the charge-transfer dynamics obtained above for the system IA (c) and IB (d).

mainly results in an expected hyperchromic effect, increasing the absorbance for this pyrene peak.

The charge transfer profiles obtained from an Ehrenfest dynamics at $0 \mathrm{~K}$ are presented in Fig. 7e. Changes in the Mulliken charges with respect to their ground-state values as a function of time are shown for IIB and IIB', with the GNC contribution and the two pyrenes derivative contributions (altogether) detailed. A charge transfer from the GNC (positive $\Delta q$ ) to the two pyrene units (negative $\Delta q$ ) is observed. In both cases appears a charge separation after $100 \mathrm{fs}$, and the ultrafast nature of the tranfer is again found here, with an stable charge separation at longer timescales. Compared to IB, the amount of charge transfer is roughly two-times greater in IIB (a bit more) and in IIB' (a bit less), showing that the "increased transfer" effect expected when adding a second fluorophore unit is actually obtained, but also depends on the pyrene positions. This higher amount of charge transferred for IIB can be tracked in the charge contribution analysis in Fig. S16 of the Supporting Information. It reveals that, when the two pyrene units are attached at each side of the GNC in IIB', both of the pyrene present a lower acceptor character compared to the unique pyrene in IB. Meanwhile in the IIB system, when the two pyrene units are anchored on neighbor sulfurs accept, only the acceptor character of one pyrene is reduced, while the second unit accept an amount of charge superior to the one of the sole pyrene in IB. These observations hint to the conclusion that uni-directional electron transfer in IIB is more efficient in such functionalized devices, than having to 'spread' the charges at both sides of the system like in IIB'.

These findings highlight, in a first approach, and at least for the systems under study, the importance of the number of chromophores for the charge transfer process. In a more detailed approach, the choice of grafting site for the molecule is an other 


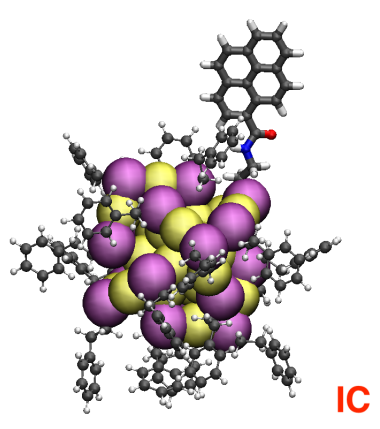

(a)

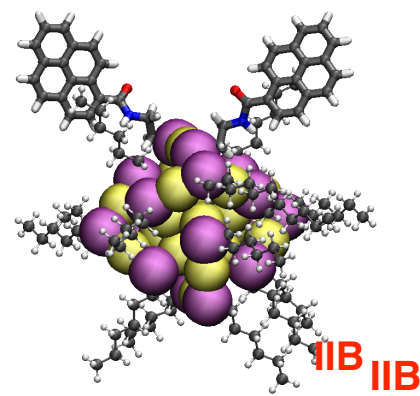

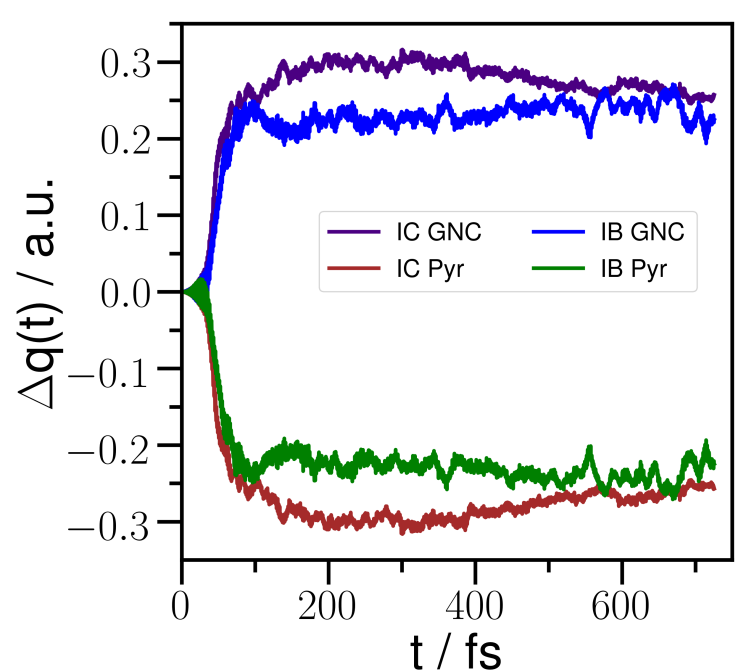

(b)

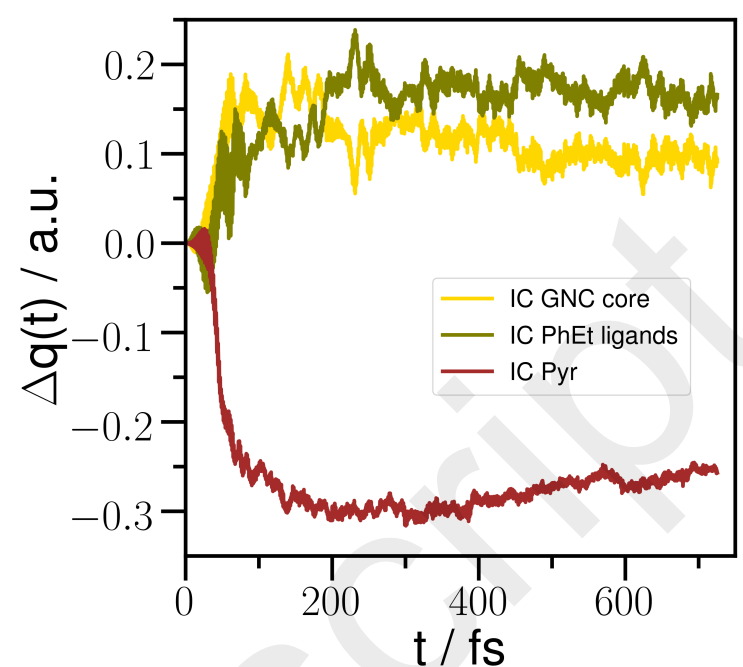

(c)

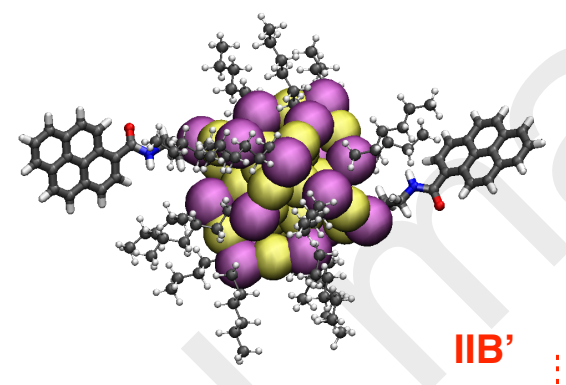

IIB'

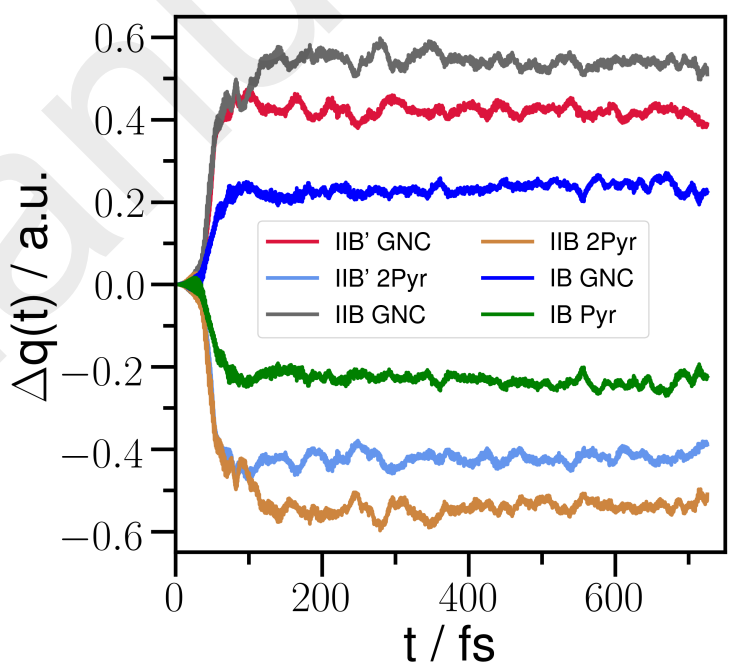

(e)

(d)

Fig. 7 (a) IC system (see Fig. 2 for color codes of atoms) (b) $\Delta \mathrm{q}$ as a function of time for the IC system.(c) Contribution analysis for the $\Delta \mathrm{q}$ as a function of time for the IC system. (d) IIB and IIB' systems (e) $\Delta \mathrm{q}$ as a function of time for the IIB and IIB' systems and compared with the IB study case.

criterion for optimization of the photoinduced event.

\section{Conclusions}

A computational modeling study of an experimental nano-sized hybrid system consisting in an hexyl-protected GNC functionalized with a pyrene fluorophore unit is presented. The theoretical results reported are in accordance with the spectroscopic findings of M.S. Devadas et al. ${ }^{9}$ suggesting that the fast and computationally cheap Ehrenfest dynamics based on TD-DFTB used here is appropriate for describing the photoinduced events in such complex systems. In short, an ultrafast charge-transfer process is observed under excitation, with a consequent amount of charge separation. The system reaches a stationary state with a charge transferred from the GNC to the pyrene derivative, supporting the GNC electron donor nature experimentally observed for this system.
For the system IB, the presence of hexyl protecting ligands around the GNC core is the main criterion to obtain a stable charge-transfer state. In this case it is possible to propose a three steps mechanism. First, at early stages ( $<50 \mathrm{fs}$ ), the dye is being excited. Then, in a second step, a small amount of charge is transferred directly from the GNC metallic core to the pyrene residue until, in a third step, the $\mathrm{C}_{6} \mathrm{H}_{13}$ ligands stabilize the charge transfer by donating to the GNC core and pyrene residue. Our results demonstrate the critical role of the protecting ligands in the ultrafast charge transfer mechanism. In particular, the importance of coherent vibronic couplings in the process is highlighted. Further research needs to be done in order to get more insights about the nature of these couplings and how can we take advantage of them to improve the electron transfer.

The nature of the protecting ligands does not in fine modify 
the light-induced charge separation outcome, but do modify the mechanism of charge transfer, with pyrene residue acting as acceptor in any case, and the GNC core acting either as a donor or a second acceptor. Increasing the number of chromophores in the systems does increase the amount of charge transferred, with the position of the different fluorophores attached being a sub-criterion for controlling the charge transfer process.

Such atomistic rationalization of the photoinduced electron flow will undoubtedly serve as useful guidelines for designing gold-organics opto-electronic devices at the nanoscale. In addition, the success of the computational approach employed in this work opens the door for further theoretical and joint theoreti$\mathrm{cal} /$ experimental studies on similar or larger systems with interest for photochemistry and nanoscience supported by the advantages of using a non-expensive quantum chemistry method.

\section{Conflicts of interest}

The authors declare no conflict of interest.

\section{Acknowledgements}

ADC would like to thank to the RTG-QM ${ }^{3}$ Program for the doctoral fellowship and to the high-computing resources of the BCCMS, University of Bremen. This work was supported by the ANR FALCON project, grant ANR-20-CE09-0002-01 of the French Agence Nationale de la Recherche.

\section{Notes and references}

1 Y. Du, H. Sheng, D. Astruc and M. Zhu, Chem. Rev., 2020, 120, 526-622.

2 W. Ding, C. Huang, L. Guan, X. Liu, Z. Luo and W. Li, Chem. Phys. Lett., 2017, 676, 18 - 24.

3 M. Zhu, C. M. Aikens, F. J. Hollander, G. C. Schatz and R. Jin, J. Am. Chem. Soc., 2008, 130, 5883-5885.

4 H. Qian and R. Jin, Nano Lett., 2009, 9, 4083-4087.

5 N. A. Sakthivel, S. Theivendran, V. Ganeshraj, A. G. Oliver and A. Dass, J. Am. Chem. Soc., 2017, 139, 15450-15459.

6 C. Kumara, X. Zuo, J. Ilavsky, D. Cullen and A. Dass, J. Phys. Chem. C, 2015, 119, 11260-11266.

7 M. A. Abbas, P. V. Kamat and J. H. Bang, ACS Energy Letters, 2018, 3, 840-854.

8 Y. Negishi, U. Kamimura, M. Ide and M. Hirayama, Nanoscale, 2012, 4, 4263-4268.

9 M. S. Devadas, K. Kwak, J.-W. Park, J.-H. Choi, C.-H. Jun, E. Sinn, G. Ramakrishna and D. Lee, J. Phys. Chem. Lett., 2010, 1, 1497-1503.

10 B. Varnholt, R. Letrun, J. J. Bergkamp, Y. Fu, O. Yushchenko, S. Decurtins, E. Vauthey, S.-X. Liu and T. Burgi, Phys. Chem. Chem. Phys., 2015, 17, 14788-14795.

11 A. Fihey, F. Maurel and A. Perrier, J. Phys. Chem. C, 2014, 118, 4444-4453.

12 C. Azarias, C. Adamo and A. Perrier, Phys. Chem. Chem. Phys., 2016, 18, 7737-7750.

13 V. Schwanen and F. Remacle, Nano Letters, 2017, 17, 56725681.

14 K. L. D. M. Weerawardene, P. Pandeya, M. Zhou, Y. Chen,
R. Jin and C. M. Aikens, J. Am. Chem. Soc., 2019, 141, 1871518726.

15 R. D. Senanayake, A. V. Akimov and C. M. Aikens, J. Phys. Chem. C, 2017, 121, 10653-10662.

16 X. Chen, O. V. Prezhdo, Z. Ma, T. Hou, Z. Guo and Y. Li, Phys. Status Solidi B, 2016, 253, 458-462.

17 M. Elstner, D. Porezag, G. Jungnickel, J. Elsner, M. Haugk, T. Frauenheim, S. Suhai and G. Seifert, Phys. Rev. B, 1998, 58, 7260-7268.

18 B. Aradi, B. Hourahine and T. Frauenheim, Journal of Physical Chemistry A, 2007, 111, 5678-5684.

19 B. Hourahine, B. Aradi, V. Blum, F. Bonafé, A. Buccheri, C. Camacho, C. Cevallos, M. Y. Deshaye, T. Dumitrică, A. Dominguez, S. Ehlert, M. Elstner, T. van der Heide, J. Hermann, S. Irle, J. J. Kranz, C. Köhler, T. Kowalczyk, T. Kubař, I. S. Lee, V. Lutsker, R. J. Maurer, S. K. Min, I. Mitchell, C. Negre, T. A. Niehaus, A. M. N. Niklasson, A. J. Page, A. Pecchia, G. Penazzi, M. P. Persson, J. Řezáč, C. G. Sánchez, M. Sternberg, M. Stöhr, F. Stuckenberg, A. Tkatchenko, V. W.-z. Yu and T. Frauenheim, The Journal of Chemical Physics, 2020, 152, 124101.

20 T. A. Niehaus, S. Suhai, F. Della Sala, P. Lugli, M. Elstner, G. Seifert and T. Frauenheim, Phys. Rev. B, 2001, 63, 085108.

21 O. A. Douglas-Gallardo, M. Berdakin and C. G. Sánchez, J. Phys. Chem. C, 2016, 120, 24389-24399.

22 F. P. Bonafé, B. Aradi, B. Hourahine, C. R. Medrano, F. J. Hernández, T. Frauenheim and C. G. Sánchez, J. Chem. Theory Comput., 2020, 16, 4454-4469.

23 M. B. Oviedo, C. F. a. Negre and C. G. Sánchez, Physical chemistry chemical physics : PCCP, 2010, 12, 6706-11.

24 M. B. Oviedo and C. G. Sánchez, Journal of Physical Chemistry A, 2011, 115, 12280-12285.

25 M. Berdakin, M. I. Taccone, G. A. Pino and C. G. Sánchez, Phys. Chem. Chem. Phys., 2017, 19, 5721-5726.

26 M. Berdakin, M. I. Taccone, G. A. Pino and C. G. Sánchez, Physical Chemistry Chemical Physics, 2017, 19, 5721-5726.

27 C. F. Negre and C. G. Sánchez, Chemical Physics Letters, 2010, 494, 255-259.

28 O. A. Douglas-Gallardo, M. Berdakin, T. Frauenheim and C. G. Sánchez, Nanoscale, 2019, 11, 8604-8615.

29 M. Berdakin, O. A. Douglas-Gallardo and C. G. Sánchez, Journal of Physical Chemistry C, 2020, 124, 1631-1639.

30 Nanoscale, 2017, 9, 17471-17480.

31 C. Mansilla Wettstein, F. P. Bonafé, M. B. Oviedo and C. G. Sánchez, J. Chem. Phys., 2016, 144, 224305.

32 F. P. Bonafé, B. Aradi, B. Hourahine, C. R. Medrano, F. J. Hernández, T. Frauenheim and C. G. Sánchez, Journal of Chemical Theory and Computation, 2020, 16, 4454-4469.

33 C. F. a. Negre, V. C. Fuertes, M. B. Oviedo, F. Y. Oliva and C. G. Sánchez, The Journal of Physical Chemistry C, 2012, 116, 14748-14753.

34 M. B. Oviedo, X. Zarate, C. F. A. Negre, E. Schott, R. ArratiaPérez and C. G. Sánchez, The Journal of Physical Chemistry Letters, 2012, 3, 2548-2555. 
35 V. C. Fuertes, C. F. a. Negre, M. B. Oviedo, F. P. Bonafé, F. Y. Oliva and C. G. Sánchez, Journal of physics. Condensed matter : an Institute of Physics journal, 2013, 25, 115304.

36 C. Mansilla Wettstein and C. G. Sánchez, Physical Chemistry Chemical Physics, 2018, 20, 21910-21916.

37 D. M. Marquez and C. G. Sánchez, Physical Chemistry Chemical Physics, 2018, 20, 26280-26287.

38 C. R. Medrano, M. B. Oviedo and C. G. Sánchez, Phys. Chem. Chem. Phys., 2016, 18, 14840-14849.

39 C. R. Medrano and C. G. Sánchez, The Journal of Physical Chemistry Letters, 2018, 3517-3524.

40 C. A. Rozzi, S. M. Falke, N. Spallanzani, A. Rubio, E. Molinari, D. Brida, M. Maiuri, G. Cerullo, H. Schramm, J. Christoffers and C. Lienau, Nature communications, 2013, 4, 1602.

41 S. M. Falke, C. A. Rozzi, D. Brida, M. Maiuri, M. Amato, E. Sommer, A. De Sio, A. Rubio, G. Cerullo, E. Molinari and C. Lienau, Science (New York, N.Y.), 2014, 344, 1001-1005.

42 A. De Sio, F. Troiani, M. Maiuri, J. Réhault, E. Sommer, J. Lim, S. F. Huelga, M. B. Plenio, C. A. Rozzi, G. Cerullo, E. Molinari and C. Lienau, Nature Communications, 2016, 7, 1-8.

43 R. Long and O. V. Prezhdo, Nano Letters, 2016, 16, 19962003.

44 Q. Zheng, W. A. Saidi, Y. Xie, Z. Lan, O. V. Prezhdo, H. Petek and J. Zhao, Nano Letters, 2017, 17, 6435-6442.

45 F. P. Bonafé, B. Aradi, M. Guan, O. A. Douglas-Gallardo, C. Lian, S. Meng, T. Frauenheim and C. G. Sánchez, Nanoscale., 2017, 9, 12391-12397.

46 F. P. Bonafé, F. J. Hernández, B. Aradi, T. Frauenheim and C. G. Sánchez, J. Phys. Chem. Lett., 2018, 9, 4355-4359.

47 F. J. Hernández, F. P. Bonafé, B. Aradi, T. Frauenheim and C. G. Sánchez, Journal of Physical Chemistry A, 2019, 123, 2065-2072.

48 M. Elstner, D. Porezag, G. Jungnickel, J. Elsner, M. Haugk, T. Frauenheim, S. Suhai and G. Seifert, Physical Review B, 1998, 58, 7260-7268.

49 M. Elstner and G. Seifert, 2014.

50 A. S. Christensen, T. Kubař, Q. Cui and M. Elstner, Chemical Reviews, 2016, 116, 5301-5337.

51 M. Gaus, Q. Cui and M. Elstner, Wiley Interdisciplinary Reviews: Computational Molecular Science, 2014, 4, 49-61.

52 B. Hourahine, B. Aradi, V. Blum, F. Bonafé, A. Buccheri, C. Camacho, C. Cevallos, M. Y. Deshaye, T. Dumitrică, A. Dominguez, S. Ehlert, M. Elstner, T. van der Heide, J. Hermann, S. Irle, J. J. Kranz, C. Köhler, T. Kowalczyk, T. Kubař, I. S. Lee, V. Lutsker, R. J. Maurer, S. K. Min, I. Mitchell, C. Negre, T. A. Niehaus, A. M. N. Niklasson, A. J. Page, A. Pecchia, G. Penazzi, M. P. Persson, J. Řezáč, C. G. Sánchez, M. Sternberg, M. Stöhr, F. Stuckenberg, A. Tkatchenko, V. W.-z. Yu and
T. Frauenheim, J. Chem. Phys., 2020, 152, 124101.

53 A. Fihey, C. Hettich, J. Touzeau, F. Maurel, A. Perrier, C. Köhler, B. Aradi and T. Frauenheim, J. Comput. Chem., 2015, 36, 2075-2087.

54 M. B. Oviedo and C. G. Sánchez, J. Phys. Chem. A, 2011, 115, 12280 .

55 C. F. A. Negre, V. C. Fuertes, M. B. Oviedo, F. Y. Oliva and C. G. Sánchez, J. Phys. Chem. C, 2012, 116, 14748.

56 M. B. Oviedo, X. Zarate, C. F. a. Negre, E. Schott, R. ArratiaPérez and C. G. Sánchez, J. Phys. Chem. Lett., 2012, 3, 2548.

57 O. A. Douglas-Gallardo, M. Berdakin and C. G. Sánchez, J. Phys. Chem. C, 2016, 120, 24389.

58 C. R. Medrano, M. B. Oviedo and C. G. Sánchez, Phys. Chem. Chem. Phys., 2016, 18, 14840.

59 M. J. Frisch, G. W. Trucks, H. B. Schlegel, G. E. Scuseria, M. A. Robb, J. R. Cheeseman, G. Scalmani, V. Barone, G. A. Petersson, H. Nakatsuji, X. Li, M. Caricato, A. V. Marenich, J. Bloino, B. G. Janesko, R. Gomperts, B. Mennucci, H. P. Hratchian, J. V. Ortiz, A. F. Izmaylov, J. L. Sonnenberg, D. WilliamsYoung, F. Ding, F. Lipparini, F. Egidi, J. Goings, B. Peng, A. Petrone, T. Henderson, D. Ranasinghe, V. G. Zakrzewski, J. Gao, N. Rega, G. Zheng, W. Liang, M. Hada, M. Ehara, K. Toyota, R. Fukuda, J. Hasegawa, M. Ishida, T. Nakajima, Y. Honda, O. Kitao, H. Nakai, T. Vreven, K. Throssell, J. A. Montgomery, Jr., J. E. Peralta, F. Ogliaro, M. J. Bearpark, J. J. Heyd, E. N. Brothers, K. N. Kudin, V. N. Staroverov, T. A. Keith, R. Kobayashi, J. Normand, K. Raghavachari, A. P. Rendell, J. C. Burant, S. S. Iyengar, J. Tomasi, M. Cossi, J. M. Millam, M. Klene, C. Adamo, R. Cammi, J. W. Ochterski, R. L. Martin, K. Morokuma, O. Farkas, J. B. Foresman and D. J. Fox, Gaussian 16 Revision C.01, 2016, Gaussian Inc. Wallingford CT.

60 A. Nitzan, Chemical Dynamics in Condensed Phases: Relaxation, Transfer, and Reactions in Condensed Molecular Systems, Oxford University Press, 2006, pp. 668-670.

61 V. Q. Vuong, J. Akkarapattiakal Kuriappan, M. Kubillus, J. J. Kranz, T. Mast, T. A. Niehaus, S. Irle and M. Elstner, J. Chem. Theory Comput., 2018, 14, 115-125.

62 M. Zhu, C. M. Aikens, F. J. Hollander, G. C. Schatz and R. Jin, Journal of the American Chemical Society, 2008, 130, 58835885.

63 S. Yamazoe, K. Koyasu and T. Tsukuda, Accounts of Chemical Research, 2014, 47, 816-824.

64 N. Sheppard, Trans. Faraday Soc., 1950, 46, 429-439.

65 E. J. Bastian and R. B. Martin, J. Phys. Chem., 1973, 77, 11291133.

66 B. Varnholt, P. Oulevey, S. Luber, C. Kumara, A. Dass and T. Bürgi, J. Phys. Chem. C, 2014, 118, 9604-9611.

67 T. Bürgi, Nanoscale, 2015, 7, 15553-15567. 